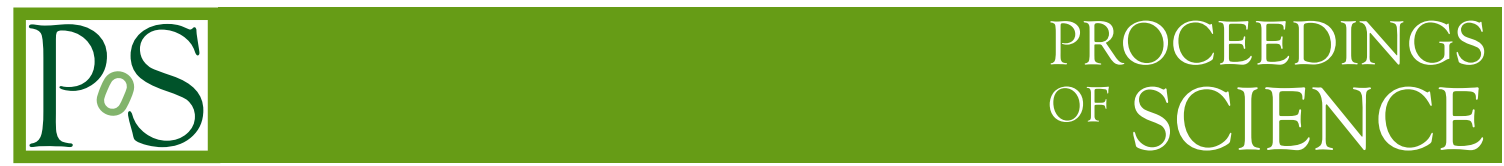

\title{
New experimental results on light and heavy hadrons
}

\author{
Chang-Zheng Yuan ${ }^{a, b, *}$ \\ ${ }^{a}$ Institute of High Energy Physics, Chinese Academy of Sciences, \\ 19B Yuquan Road, Beijing, China \\ ${ }^{b}$ University of Chinese Academy of Sciences, \\ 19A Yuquan Road, Beijing, China \\ E-mail: yuancz@ihep.ac.cn
}

We review the experimental progress on the study of light and heavy hadrons and focus on recent discoveries of the XYZ states, pentaquark states, $T_{c s}$ and $T_{c c}$ states in the heavy hadron sector, and on the $\pi_{1}(1600)$ and the states close to the $p \bar{p}$ threshold in the light hadron sector. The observations suggest that we did observe hadronic molecules and we also observed hadronic states with some other quark configurations.

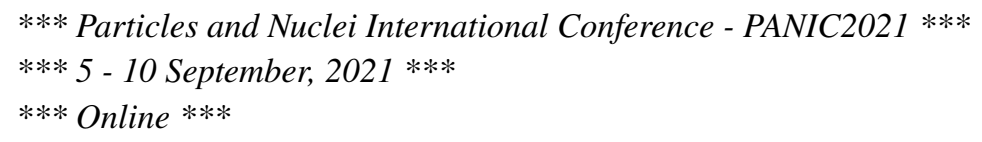

${ }^{*}$ Speaker 


\section{Introduction}

Hadron spectroscopy is a field of frequent discoveries and surprises, and the theoretical difficulties in understanding the strong interaction in the color-confinement regime make the field even more fascinating. The tremendous data collected by the BaBar, Belle, BESIII, COMPASS, LHCb, and other experiments and improved theoretical tools developed to analyze the experimental data result in rapid progress of the field.

In this talk, we review the progress in both heavy hadrons and light hadrons, and we focus on those states with exotic properties, including the quarkoniumlike states, also known as the XYZ states, pentaquark states, and light hadrons close to thresholds or with exotic quantum numbers.

\section{New results on the heavy hadrons}

In the conventional quark model, mesons are composed of one quark and one anti-quark, while baryons are composed of three quarks. However, many quarkoniumlike states were discovered at two $B$-factories BaBar and Belle [1] in the first decade of the 21st century. Whereas some of these are good candidates of quarkonium states, many other states have exotic properties, which may indicate that exotic states, such as multi-quark state, hadronic molecule, or hybrid, have been observed [2].

BaBar and Belle experiments finished their data taking in 2008 and 2010, respectively, and the data are still used for various physics analyses. BESIII [3] and LHCb [4] experiments started data taking and contributed to the study of the XYZ particles, pentaquark states, and other heavy hadrons. Most of the discoveries of the such states were made at these four experiments.

Figure 1 shows the history of the discovery of the heavy exotic states, started from the observation of the $X$ (3872) in 2003 [5]. It seems to us that the new spectrum has emerged although more effort is need to understand the exact nature of them. We show some recent experimental results on these particles.

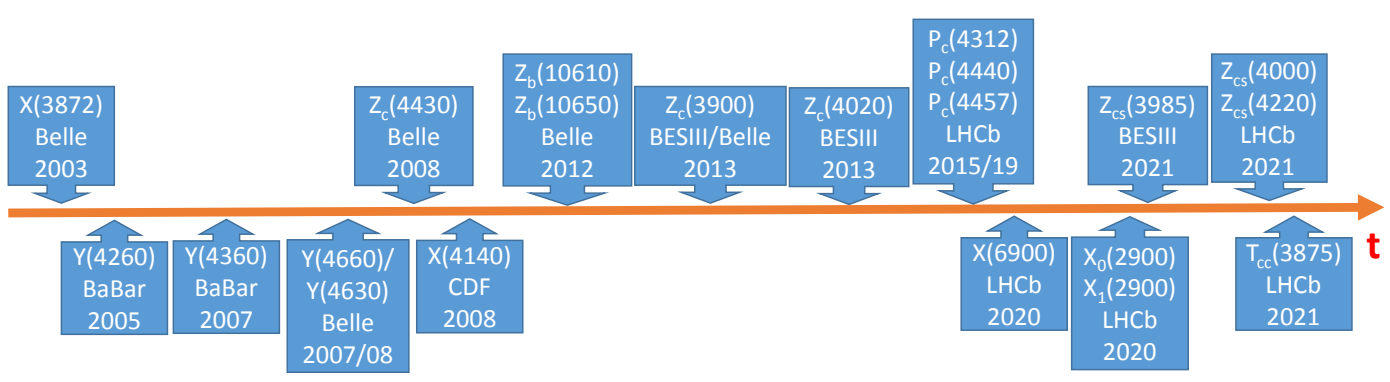

Figure 1: Discovery of heavy exotic states from experiments.

\subsection{The $X(3872)$}

The $X(3872)$ was observed in 2003 by the Belle experiment [5], and it was confirmed later by CDF [6] and $D 0$ [7] experiments in $p \bar{p}$ collision. After almost 20 years' study, we know this state much better than any of the other similar states. 
The mass of the $X(3872)$ has been measured as $3871.65 \pm 0.06 \mathrm{MeV}$ [8], which is lower than the mass threshold of $\bar{D}^{0} D^{* 0}, 3871.69 \pm 0.11 \mathrm{MeV}$, by $0.04 \pm 0.12 \mathrm{MeV}$, to be compared with the bounding energy of the deuteron of $2.2 \mathrm{MeV}$. If the $X(3872)$ is a molecule of $\bar{D}^{0} D^{* 0}$, its size will be larger than $5 \mathrm{fm}$, much larger than the size of a typical hadron.

The width measurements are less precise and model dependent since the $X(3872)$ is very narrow and the mass resolution of the experiments is usually much larger than the intrinsic width. Fitting the $\pi^{+} \pi^{-} J / \psi$ invariant mass distribution with a Breit-Wigner function, LHCb reported a width of about $1 \mathrm{MeV}$ (the mass resolution is $2.4-3.0 \mathrm{MeV}$ ); and the fit with a Flatte function with constraints from other measurements yields a FWHM of $0.22 \mathrm{MeV}$ which depends strongly on the $X(3872) \rightarrow \bar{D}^{0} D^{* 0}$ coupling $[9,10]$. Although the statistics are low at BESIII experiments, the high efficiencies of reconstructing all the $X(3872)$ decays modes and the very good mass resolution in the $\bar{D}^{0} D^{* 0}$ mode (<1 MeV) make it possible to measure the line shape of the $X(3872)$ state [11].

The production of the $X(3872)$ has been reported in many different kinds of processes, in $B$ and $B_{s}$ meson decays, in $\Lambda_{b}$ baryon decays, in $p \bar{p}$ and $p p$ collisions, and in $e^{+} e^{-}$annihilation [12]. Recently, evidence for $X(3872)$ production in $P b P b$ and two-photon collisions is reported.

CMS experiment reported a $4.2 \sigma$ signal of the $X(3872)$ in $P b P b$ collision at $5.02 \mathrm{TeV}$ [13], and it is interesting to note that its production rate relative to the $\psi(2 S)$ is much larger than in the $p p$ collision at 7 and $8 \mathrm{TeV}$, although the uncertainty is large. If this is confirmed, this is a supplemental information to understand the nature of this state.

Belle experiment searched for the $X(3872)$ in $\gamma \gamma^{*}$ fusion [14]. Three events are observed in the signal region, corresponds to a statistical significance of $3.2 \sigma$. Since we know that the $X(3872)$ has $J^{P C}=1^{++}$, it cannot be produced in two real-photon collision, the production requires at least one of the photons is virtual.

The total production rate of the $X(3872)$ in $B$ decays was measured by reconstructing a $B^{-}$and a charged kaon from $B^{+}$decays and checking the recoiling mass of the $B^{-} K^{+}$system. BaBar observed a small peak, corresponding to a $3.0 \sigma$ significance at the $X(3872)$ signal region, and measured the branching fraction of $B^{+} \rightarrow K^{+} X(3872)$ as $(2.1 \pm 0.6 \pm 0.3) \times 10^{-4}$ [15]. Belle did the same analysis, but the signal is less significant and the resulting branching fraction is $(1.2 \pm 1.1 \pm 0.1) \times 10^{-4}$ and the signal significance is $1.1 \sigma$ [16]. Although the signals are not very significant, we know this process must exist because this state has been observed in its many exclusive decays. One can use these measurements combined with other information, such as the product branching fractions and the ratio of the branching fractions, to determine the decay branching fractions of the $X(3872)$, including its decays to open charm final states, hadronic transitions, and radiative transitions. There could be a small branching fraction to light hadrons, but no experiment has observed any of them.

The authors of Ref. [17] did a global fit to the currently available experimental measurements of the product branching fractions and the ratios of the branching fractions. It is found that the branching fraction of open charm decay is around 50\% and that of each hadronic transition is at a few per cent level, there is still around one-third of the $X(3872)$ decays unknown. This should be searched for in the future experiments like BESIII and Belle II.

One still very confusing decay mode is $X(3872) \rightarrow \gamma \psi(2 S)$. There have been four different measurements. The BaBar experiment claimed $3.5 \sigma$ evidence of this mode and a production rate relative to $X(3872) \rightarrow \gamma J / \psi$ is $3.4 \pm 1.4$ [18], but Belle failed to find significant signal and the ratio was measured to be less than 2.1 at the $90 \%$ C.L. [19]. Three years later, LHCb did the 
same analysis and the found a $4.4 \sigma$ signal with a ratio of $2.46 \pm 0.81$ [20], but a recent BESIII measurement found no signal and a much stringent upper limit of the ratio is determined to be 0.59 at the $90 \%$ C.L. [11]. So we have four experiments here, two claimed evidence and the other two observed nothing. So it is still not clear whether this channel, $X(3872) \rightarrow \gamma \psi(2 S)$, exists or if it exists, how small the branching fraction is.

\subsection{The $Y$ states}

The $Y$ states were discovered in the initial state radiation in the $B$-factory experiments, and they have $J^{P C}=1^{--}$. So these state can also be produced directly in $e^{+} e^{-}$annihilation experiment like BESIII. In this case, much larger statistics are achieved and these states, the $Y(4260), Y(4360)$, $Y(4660)$, and so on, are measured with improved precision.

The $Y$ (4260) was observed in 2005 by BaBar experiment [21] and the most precise measurement is from the BESIII experiment [22]. By doing a high luminosity energy scan in the vicinity of the $Y(4260)$, BESIII found the peak of the $Y(4260)$ is much lower (so now named the $Y(4220)$ ) than that from previous measurements and the width is narrow, and there is a high mass shoulder with a mass of $4.32 \mathrm{GeV}$ if fitted with a BW function. Since then, more new decay modes of the $Y(4220)$ were observed including $\pi^{+} \pi^{-} h_{c}, \omega \chi_{c 0}$, and $\pi \bar{D} D^{*}+c . c$.

A global fit [23] to these four modes was performed, and One determines the mass of the $Y(4220)$ as $4220 \pm 6 \mathrm{MeV}$ and the width of $56 \pm 8 \mathrm{MeV}$. It is interesting to point out that the mass of this resonance is quite close to the threshold of threshold of $D_{s}^{*+} D_{s}^{*-}$ which is $4224 \mathrm{MeV}$. Since there are more $Y(4220)$ decay modes observed $\left(\pi^{+} \pi^{-} \psi(2 S), \eta_{c} \pi^{+} \pi^{-} \pi^{0}\right.$, and so on), this combined fit can be updated with more information.

For the state at $4.66 \mathrm{GeV}$, it was observed in $e^{+} e^{-} \rightarrow \pi^{+} \pi^{-} \psi(2 S)$ by Belle [24], and confirmed by BaBar [25]. The peak position is at around $4.66 \mathrm{GeV}$, thus it is called the $Y(4660)$. There is another state observed in $e^{+} e^{-} \rightarrow \Lambda_{c} \bar{\Lambda}_{c}$ by the Belle experiment [26], but the peak is at around $4.63 \mathrm{GeV}$, although the error is large. It is not clear whether these two states are the same or whether there are two states in this energy region.

BESIII data on $e^{+} e^{-} \rightarrow \pi^{+} \pi^{-} \psi(2 S)$ mode from 4 to $4.7 \mathrm{GeV}$ confirmed the Belle and BaBar observations with much improved precision [27], BESIII has data now covering from threshold to $4.95 \mathrm{GeV}$, comparable precision as at $4.6 \mathrm{GeV}$ is expected at high energies, so we expect better measurement of the $Y(4660)$ state from BESIII soon.

Belle reported measurements of two open-charm final states. There is a very beautiful peak observed at around $4.63 \mathrm{GeV}$ in $D_{s}^{+} D_{s 1}(2536)^{-}+$c.c. mode and the signal significance is $5.9 \sigma$ [28]. The signal in $D_{s}^{+} D_{s 2}(2573)^{-}+$c.c. mode is not so significant, is only $3.4 \sigma$ [29].

If we put all these information together, we can find that the peak position is about $4.65 \mathrm{GeV}$ in $\pi^{+} \pi^{-} \psi(2 S)$ mode, and that in open charm baryon and meson pair final states is below $4.65 \mathrm{GeV}$, There are differences from different final states. We need more information to really understand the structures in this mass region.

\subsection{Charged quarkonium states}

These include the $Z_{c}, Z_{b}$, and also the $Z_{c s}$ states. Since these states decay into final states with one pair of heavy quarks and charged, there must be at least four quarks in their configuration. 
The $Z_{c}$ (3900) discovered by BESIII [30] and Belle [31] is quite close to the $\bar{D} D^{*}$ threshold, and the $Z_{c}(4020)$ discovered by BESIII is quite close to the $\bar{D}^{*} D^{*}$ threshold [32], while the $Z_{c}(4430)$ discovered by the Belle [33] is not quite close to any of the open charm threshold. In the bottom sector, The $Z_{b}(10610)$ and $Z_{b}(10650)$ discovered by Belle [34] are close to the $\bar{B} B^{*}$ and $\bar{B}^{*} B^{*}$ thresholds, respectively.

These states have been observed for some time. Recent studies try to search for states with one of the four quarks replaced by a different quark, for example, the $Z_{c s}$ states with quark content $c \bar{c} u \bar{s}$. There are three different measurements, one from Belle in $e^{+} e^{-} \rightarrow K^{+} K^{-} J / \psi$ [35], another from BESIII in $e^{+} e^{-} \rightarrow K\left(D D_{s}^{*}+D^{*} D_{s}\right)$ [36], and the third from $\mathrm{LHCb}$ in $B^{+} \rightarrow J / \psi K^{+} \phi$ [37].

No significant signal was observed in Belle data due to low statistics of the process produced via ISR. BESIII announced observation of a near-threshold structure $Z_{c s}(3985)$ in the $K^{+}$recoil-mass spectra in $e^{+} e^{-} \rightarrow K^{+}\left(D_{s}^{-} D^{* 0}+D_{s}^{*-} D^{0}\right)$ [36] with a mass of $3983 \mathrm{MeV}$ and a width of about $10 \mathrm{MeV}$; and LHCb reported two resonances decaying into $K^{ \pm} J / \psi$, the $Z_{c s}(4000)$ with a mass of $4003 \mathrm{MeV}$ and a width of about $131 \mathrm{MeV}$, and the $Z_{c s}(4220)$ with a mass of $4216 \mathrm{MeV}$ and a width of about $233 \mathrm{MeV}$ [37]. The widths of the $Z_{c s}(3985)$ and $Z_{c s}(4000)$ are quite different, so they could not be the same state. Maybe one of them is the strange partner of the $Z_{c}(3900)$ with the $d$ quark replaced with an $s$ quark.

\subsection{Pentaquark states}

In the decay of $\Lambda_{b} \rightarrow J / \psi p K^{-}$analyzed by the LHCb experiments, there are three very narrow peaks in the invariant mass distribution of $J / \psi p$ [38]. In a simple fit to the invariant mass spectrum, the resonance parameters of the $P_{c}(4312)^{+}, P_{c}(4440)^{+}$, and $P_{c}(4457)^{+}$are determined. They are all narrow, and the $P_{c}(4312)^{+}$state peaks right below the $\Sigma_{c}^{+} \bar{D}^{0}$ threshold, the $P_{c}(4457)^{+}$state peaks right below the $\Sigma_{c}^{+} \bar{D}^{* 0}$ threshold, while the $P_{c}(4457)^{+}$state peaks about $20 \mathrm{MeV}$ below it. Since the statistics are large and these structures are quite narrow, partial wave analysis (PWA) to the data sample is still not available, and the spin-parities are not known yet.

Being so close to the thresholds, they are very good candidates for the molecules of a charmed baryon and an anti-charmed meson, and we expect more similar states close to the other baryonmeson thresholds.

\subsection{States with four flavors $\left(T_{c s}\right)$ or two heavy quarks $\left(T_{c c}\right)$}

States with four different flavors have been search for and evidence $(3.9 \sigma)$ for two states $\left(X_{0}(2900)\right.$ and $X_{1}(2900)$ ) in $D^{-} K^{+}$system was reported from a PWA of $B^{+} \rightarrow D^{+} D^{-} K^{+}$events by the LHCb experiment [39]. These states, if confirmed, will be manifestly exotic, charm-strange $T_{c s}$ states with quark content $\bar{c} d u \bar{s}$.

LHCb also reported new observation of a state with two charmed quarks, $T_{c c}$ (3875) with a mass a few hundred $k \mathrm{eV}$ lower than the $D^{0} D^{*+}$ threshold and a width much less than $1 \mathrm{MeV}$ [40]. If it is interpreted as a molecule of two charmed mesons, its size will be around $7.5 \mathrm{fm}$, pretty large than a normal hadrons of $1 \mathrm{fm}$.

\subsection{Summary on heavy hadrons}

If we summarize these heavy hadrons, what we find is that some of them are quite close to the thresholds of two heavy objects, either two heavy flavor mesons or one heavy flavor 
meson and one heavy flavor baryon, like the $X(3872)\left(\bar{D}^{0} D^{* 0}\right), Y(4220)\left(D_{s}^{*+} D_{s}^{*-}\right.$ or $\left.\bar{D} D_{1}\right)$, $Z_{c}(3900)^{+}\left(\bar{D}^{0} D^{*+}\right), Z_{c}(4020)^{+}\left(\bar{D}^{* 0} D^{*+}\right), Z_{c s}^{+}\left(\bar{D}^{0} D_{s}^{*+}\right), Z_{b}(10610)^{+}\left(\bar{B}^{0} B^{*+}\right), Z_{b}(10650)^{+}$ $\left(\bar{B}^{* 0} B^{*+}\right) P_{c}(4312)^{+}\left(\Sigma_{c}^{+} \bar{D}^{0}\right), P_{c}(4457)^{+}$and $P_{c}(4457)^{+}\left(\Sigma_{c}^{+} \bar{D}^{* 0}\right)$, and $T_{c c}(3875)^{+}\left(D^{0} D^{*+}\right)$; and some other states are not close to such thresholds, such as the $Y(4360) Y(4660), Z_{c}(4430)^{+}$, $Z_{c s}(4220)^{+}$, and $X(6900)$.

These may suggest that we did observe the hadronic molecules close to thresholds and we also observed hadronic states with some other quark configurations like compact tetraquark states and so on. At the same time, these suggest that similar dynamics will also play important role in light quark sector, and this makes the understanding of the light hadron spectroscopy even harder.

\section{Light hadron spectroscopy}

We present two selected topics on light hadrons, the $\pi_{1}(1600)$ with exotic quantum numbers and the states close to $p \bar{p}$ threshold.

\subsection{The $\pi_{1}(1600)$ from COMPASS}

The $\pi_{1}(1600)$ is a state with $J^{P C}=1^{-+}$, quantum numbers cannot reach by a $q \bar{q}$ state. A recent COMPASS analysis [41] tries to understand the tensions observed between COMPASS and previous experiments, including the BNL E852 experiment, VES experiment, and also COMPASS analysis with less waves in the PWA.

It is concluded that: 1) conflicting conclusions from previous studies can be attributed to different models and treatment of $t^{\prime}$-dependence of the amplitudes; 2) Deck model can describe data in spectral shape and $t^{\prime}$-dependence for the $J^{P C}=1^{-+}$and other amplitudes; 3) Freed-isobar fit results indicate that the $P$-wave $\pi^{+} \pi^{-}$-amplitude is dominated by $\rho(770)$ for both the $\pi_{1}(1600)$ and the non-resonant $J^{P C}=1^{-+}$amplitudes.

The final conclusion quoted from the paper is shown here: "These findings largely confirm the underlying assumptions for the isobar model used in all previous partial-wave analyses addressing the $J^{P C}=1^{-+}$amplitude." Or simply speaking, the $\pi_{1}(1600)$ really exists.

\subsection{States close to $p \bar{p}$ threshold in $J / \psi$ decays}

BESIII studies of the $X(1835) \rightarrow \eta^{\prime} \pi^{+} \pi^{-}$line shape with 1.3 billion $J / \psi$ events revealed an anomalous structure in its line shape at the $p \bar{p}$ threshold that could be equally well described as interference with a new narrow resonance that has a mass nearly equal to $2 m_{p}$ or a wide resonance with an anomalously strong coupling to the $p \bar{p}$ final state [42].

States in this mass region were also reported in $J / \psi \rightarrow \omega \eta \pi^{+} \pi^{-}$[43], $\gamma 3\left(\pi^{+} \pi^{-}\right)$[44], and $\gamma \gamma \phi$ [45], but not in $J / \psi \rightarrow \omega \eta^{\prime} \pi^{+} \pi^{-}[46]$.

\section{Summary and Perspectives}

There are a lot of progress in the experimental study of hadron spectroscopy. Spectroscopy of hadronic molecules need to be further investigated, and states formed by other dynamics may have been discovered too. Study of similar states in the light quark sector $(u, d, s)$ may reveal even richer phenomena of strong interaction. It is expected that more results will be produced by the Belle II, BESIII, COMPASS, GlueX, and LHCb experiments. 


\section{Acknowledgments}

I thank the organizers for inviting me to give this review talk. This work is supported in part by National Key Research and Development Program of China (No. 2020YFA0406300), and National Natural Science Foundation of China (NSFC, Nos. 11961141012, 11835012, and 11521505).

\section{References}

[1] A. J. Bevan et al. [BaBar and Belle], Eur. Phys. J. C 74, 3026 (2014).

[2] For recent reviews, see N. Brambilla, S. Eidelman, C. Hanhart, A. Nefediev, C. P. Shen, C. E. Thomas, A. Vairo and C. Z. Yuan, Phys. Rept. 873, 1-154 (2020); F. K. Guo, C. Hanhart, U. G. Meißner, Q. Wang, Q. Zhao and B. S. Zou, Rev. Mod. Phys. 90, no. 1, 015004 (2018). H. X. Chen, W. Chen, X. Liu and S. L. Zhu, Phys. Rept. 639, 1 (2016).

[3] C. Z. Yuan, Int. J. Mod. Phys. A 33, no. 21, 1830018 (2018).

[4] R. Aaij et al. [LHCb], Int. J. Mod. Phys. A 30, 1530022 (2015).

[5] S. K. Choi et al. [Belle], Phys. Rev. Lett. 91, 262001 (2003).

[6] D. Acosta et al. [CDF], Phys. Rev. Lett. 93, 072001 (2004).

[7] V. M. Abazov et al. [D0], Phys. Rev. Lett. 93, 162002 (2004).

[8] P. A. Zyla et al. [Particle Data Group], PTEP 2020, no.8, 083 C01 (2020).

[9] R. Aaij et al. [LHCb], Phys. Rev. D 102, no.9, 092005 (2020).

[10] R. Aaij et al. [LHCb], JHEP 08, 123 (2020).

[11] M. Ablikim et al. [BESIII], Phys. Rev. Lett. 124, 242001 (2019).

[12] M. Ablikim et al. [BESIII], Phys. Rev. Lett. 112, 092001 (2014).

[13] A. M. Sirunyan et al. [CMS], [arXiv:2102.13048 [hep-ex]].

[14] Y. Teramoto et al. [Belle], Phys. Rev. Lett. 126, no.12, 122001 (2021).

[15] J. P. Lees et al. [BaBar], Phys. Rev. Lett. 124, no.15, 152001 (2020).

[16] Y. Kato et al. [Belle], Phys. Rev. D 97, no.1, 012005 (2018).

[17] C. Li and C. Z. Yuan, Phys. Rev. D 100, no.9, 094003 (2019).

[18] B. Aubert et al. [BaBar], Phys. Rev. Lett. 102, 132001 (2009)

[19] V. Bhardwaj et al. [Belle], Phys. Rev. Lett. 107, 091803 (2011).

[20] R. Aaij et al. [LHCb], Nucl. Phys. B 886, 665 (2014). 
[21] B. Aubert et al. [BaBar], Phys. Rev. Lett. 95, 142001 (2005).

[22] M. Ablikim et al. [BESIII], Phys. Rev. Lett. 118, no.9, 092001 (2017).

[23] X. Y. Gao, C. P. Shen and C. Z. Yuan, Phys. Rev. D 95, no.9, 092007 (2017).

[24] X. L. Wang et al. [Belle], Phys. Rev. Lett. 99, 142002 (2007).

[25] J. P. Lees et al. [BaBar], Phys. Rev. D 89, no.11, 111103 (2014).

[26] G. Pakhlova et al. [Belle], Phys. Rev. Lett. 101, 172001 (2008).

[27] M. Ablikim et al. [BESIII], Phys. Rev. D 104, no.5, 052012 (2021).

[28] S. Jia et al. [Belle], Phys. Rev. D 100, no.11, 111103 (2019).

[29] S. Jia et al. [Belle], Phys. Rev. D 101, no.9, 091101 (2020).

[30] M. Ablikim et al. [BESIII], Phys. Rev. Lett. 110, 252001 (2013).

[31] Z. Q. Liu et al. [Belle], Phys. Rev. Lett. 110, 252002 (2013)

[32] M. Ablikim et al. [BESIII], Phys. Rev. Lett. 111, no.24, 242001 (2013).

[33] S. K. Choi et al. [Belle], Phys. Rev. Lett. 100, 142001 (2008).

[34] A. Bondar et al. [Belle], Phys. Rev. Lett. 108, 122001 (2012).

[35] C. P. Shen et al. [Belle], Phys. Rev. D 89, no.7, 072015 (2014).

[36] M. Ablikim et al. [BESIII], Phys. Rev. Lett. 126, no.10, 102001 (2021).

[37] R. Aaij et al. [LHCb], Phys. Rev. Lett. 127, no.8, 082001 (2021).

[38] R. Aaij et al. [LHCb], Phys. Rev. Lett. 122, no.22, 222001 (2019).

[39] R. Aaij et al. [LHCb], Phys. Rev. Lett. 125, 242001 (2020); Phys. Rev. D 102, 112003 (2020).

[40] R. Aaij et al. [LHCb], [arXiv:2109.01038 [hep-ex]]; [arXiv:2109.01056 [hep-ex]].

[41] M. G. Alexeev et al. [COMPASS], [arXiv:2108.01744 [hep-ex]].

[42] M. Ablikim et al. [BESIII], Phys. Rev. Lett. 117, no. 4, 042002 (2016).

[43] M. Ablikim et al. [BESIII], Phys. Rev. Lett. 107, 182001 (2011).

[44] M. Ablikim et al. [BESIII], Phys. Rev. D 88, no. 9, 091502 (2013).

[45] M. Ablikim et al. [BESIII], Phys. Rev. D 97, no. 5, 051101 (2018).

[46] M. Ablikim et al. [BESIII], Phys. Rev. D 99, no.7, 071101 (2019). 